\title{
AMENDMENTS
}

\section{Author Correction: Efficient electrical detection of mid-infrared graphene plasmons at room temperature}

\author{
Qiushi Guo @i Renwen Yu (D), Cheng Li, Shaofan Yuan, Bingchen Deng, F. Javier García de Abajo (i) and Fengnian Xia (D)
}

Correction to: Nature Materials https://doi.org/10.1038/s41563-018-0157-7, published online 27 August 2018.

In the version of this Article originally published, the units of the right-hand $y$ axis of Fig. 2a were incorrectly labelled as mS; they should have been $\mu$ S. Also, the $x$-axis tick marks of Fig. $3 \mathrm{~b}$ should have been aligned with Fig. 3a,c. These have now been corrected.

\section{Publisher Correction: Non-specific interactions govern cytosolic diffusion of nanosized objects in mammalian cells}

Fred Etoc, Elie Balloul, Chiara Vicario, Davide Normanno (D), Domenik Liße, Assa Sittner, Jacob Piehler (D), Maxime Dahan and Mathieu Coppey (D)

Correction to: Nature Materials https://doi.org/10.1038/s41563-018-0120-7, published online 2 July 2018.

In the version of this Article originally published, Supplementary Videos 3-5 were incorrectly labelled; 3 should have been 5, 4 should have been 3 and 5 should have been 4 . This has now been corrected.

Published online: 19 September 2018

https://doi.org/10.1038/s41563-018-0188-0

\section{Retraction Note: Large local lattice expansion in graphene adlayers grown on copper}

Chaoyu Chen DD, José Avila, Hakim Arezki, Van Luan Nguyen, Jiahong Shen, Marcin Mucha-Kruczyński, Fei Yao,

Mohamed Boutchich, Yue Chen, Young Hee Lee (D) and Maria C. Asensio

Retraction of: Nature Materials https://doi.org/10.1038/s41563-018-0053-1, published online 9 April 2018.

The authors unanimously wish to retract this Article due to their concerns about the interpretation of the low-energy electron microscopy (LEEM) and diffraction (LEED) patterns reported in the manuscript. In this study, the authors used spatial and angle-resolved photoemission spectroscopy (ARPES) to characterize graphene monolayers grown on copper foils, and observed regions of graphene adlayers with enhanced graphene/Cu interaction, higher Dirac cone doping level, moiré mini Dirac cones and large lattice expansion. All these properties have been clearly verified and reproduced by photoemission spectroscopy as well as explained by density functional theory. LEEM and LEED characterization were also carried out to confirm the existence of a moiré superlattice and lattice expansion, and the results were included in the main manuscript and Supplementary Information. On further analysis of the LEEM/LEED data, it seems that while the existence of a moiré superlattice can be corroborated, the conclusion of graphene lattice expansion (7\%) based on spatially resolved ARPES determinations cannot be confirmed by the LEEM/LEED measurements. The authors realized that these measurements were collected from statistically non-representative areas of the sample. Moreover, the fact that the raw microLEED images bear an asymmetry factor of as much as 5\% due to the instrumental aberration makes it impossible to estimate any compression or expansion of the same order. Consequently, their conclusion on the graphene lattice expansion can only be supported by the photoemission data. In view that more complete and reliable structural determinations should be conducted, all authors wish to retract this Article. 